\title{
Effects of a Five Times Sit to Stand Test on the Daily Life Independence of Korean Elderly and Cut-Off Analysis
}

\author{
Seung-Min Nam, PT, PhD • Seong-Gil Kim, PT, $\mathrm{PhD}^{1 \dagger}$ \\ Department of Physical Therapy, Daegu University \\ ${ }^{1}$ Department of Physical Therapy, Uiduk University
}

Received: September 18, 2019 / Revised: September 27, 2019 / Accepted: October 19, 2019

(C) 2019 J Korean Soc Phys Med

\section{| Abstract |}

PURPOSE: The aim of this study was to provide the standard value of the Five Times Sit to Stand Test (FTSST) measurement on the daily life independence of the elderly in Korea and examine the effects of this test on their daily lives. METHODS: This study was conducted on elderly people over 65 years of age living in Gyeongsangbuk-do, Korea. FTSST was performed while sitting position on a chair. The subjects were classified into independent and dependent living groups according to their lifestyle, and their influence was then examined through logistic regression analysis. To determine the usefulness and cut-off value of the FTSST, the analysis was performed using the ROC curve.

RESULTS: The elderly were more likely to live in a group rather than independently as the FTSST time increased $(\mathrm{p}<.05)(\mathrm{OR}=1.098)$. The area of the lower part of the ROC curve was .707, and as the FTSST increased, a subject was

$\uparrow$ Corresponding Author : Seong-Gil Kim

niceguygil@gmail.com, https://orcid.org/0000-0002-2487-5122

This is an Open Access article distributed under the terms of the Creative Commons Attribution Non-Commercial License (http://creativecommons.org/licenses/by-nc/3.0) which permits unrestricted non-commercial use, distribution, and reproduction in any medium, provided the original work is properly cited. more likely to live in a group rather than independently $(\mathrm{p}<.05)$. The cut-off value was assigned to the point where both the specificity and sensitivity were at the coordinates. The sensitivity and specificity were .626 and .753 , respectively at 15.62 seconds.

CONCLUSION: The elderly in Korea are more likely to live a group-dependent lifestyle than live independently; the likelihood of this outcome is increased further for every additional second beyond 15.62 seconds. The loss of independence of daily life could be predicted based on the status of a subject's lower leg strength using the FTSST.

Key Words: Frail Elderly, Lower Extremity, Muscle Strength, Regression Analysis, ROC Curve

\section{Introduction}

The number of elderly people in Korea has increased steadily by $3.1 \%$ annually from 1970 to 2000. In 2017, the proportion of elderly people over 65 was $13.8 \%$. The country is expected to reach a post-aged society in 2026 [1]. Therefore, as the number of elderly people in Korea increases dramatically, concerns about the quality of life and health of the elderly population are increasing. In 2011, the elderly population accounted for $32.2 \%$ of the total 
medical expenses due to the increase in their numbers [2]. In this way, medical and social expenditure as well as social interests for the elderly are increasing.

As aging generally progresses, the musculoskeletal system manifests many physical and psychological effects, such as reduced durability and flexibility, decreased exercise performance, slow recovery times of injuries due to trauma, and limited mobility and independence [3]. In addition, sensory functions begin to decrease and the functions of the organs associated with balance control, such as visual, vestibular, and proprioceptive senses are reduced, which results in increased posture sway and reduced balance [4].

This decrease in physical function causes the elderly to have less independence for daily living. Moreover this loss of functional independence increases the risk of falls and fractures due to instability during physical activities $[5,6]$, which can represent a serious hazard, resulting in a change in lifestyle where the elderly become hospitalized in either hospitals or nursing facilities. In these scenarios, it is difficult to recover the ability to perform daily activities $[7,8]$. Therefore, it is important to maintain the independence of the elderly.

In general, the leg strength is measured to evaluate the level of functional independence of the elderly indirectly. A lack of leg strength has been reported to reduce the body's stability and mobility, and limit the levels of independent daily living. Therefore, the leg strength is an important factor for predicting a deterioration of the body functions. In addition, the strength of the lower limbs predicts the risk of falling. The lower limbs play an important role in supporting the body and the ability to walk. This lowering of the lower limbs increases the risk of falling $[9,10]$. A range of devices are used to evaluate the leg strength of the elderly. On the other hand, it is difficult to evaluate accurately when the subject is an elderly person [11]. To evaluate the leg strength of the elderly, an examination method based on the activities performed in daily life has been proposed. Of the measurement tools used in clinical practice, the Five Times Sit to Stand Test (FTSST) is considered reliable and applicable to many subjects. This tool can measure the functional leg strength of the elderly and predict indirectly the aptitude to perform daily activities based on the ability to sit and stand up, which is a basic activity of everyday life and is important for functional independence [12].

On the other hand, this tool has been developed in foreign countries, and the standard value of the FTSST measurement value on daily life independence has only been presented in those contexts. Therefore, this paper provides the standard values of the FTSST measurements on the daily life independence of the elderly in Korea as well as their effects on their daily lives. These data can be used as basic data for future clinical studies of elderly people in Korea.

\section{Methods}

\section{Subjects}

This study was conducted on elderly people over 65 years of age, who were living in community and nursing homes within Gyeongsangbuk-do, Korea. This study selected 321 subjects who matched the selection criteria of the subjects. Thirty-nine people were rejected in the process of obtaining consent, and 17 were excluded during the measurement. Therefore, 265 patients (male: 71, female: 194) participated in the study. The mean age of the subjects was $78.69 \pm 7.68$ years, with a height and body weight of $155.22 \pm 8.29 \mathrm{~cm}$ and $56.18 \pm 11.40 \mathrm{~kg}$, respectively (Table 1). The subjects were 65 years of age or older and had experienced one or more falls during the past year. No serious damage to the visual or somatosensory sensations that could affect the experiment was noted; the subjects could walk more than $10 \mathrm{~m}$ by themselves. The subjects had a level of consciousness to perform this study with a MMSE-K score of 24 or higher. Before the experiment 
Table 1. General Characteristics of the Subjects

\begin{tabular}{c|c}
\hline Gender (M/F) & $71 / 194$ \\
\hline $\begin{array}{c}\text { Type of Living } \\
\text { (Community-dwelling/Nursing home) }\end{array}$ & $150 / 115$ \\
\hline Age (years) & $78.69 \pm 7.67$ \\
\hline Height (cm) & $155.18 \pm 8.27$ \\
\hline Weight (kg) & $55.98 \pm 11.66$ \\
\hline
\end{tabular}

$\mathrm{M} \pm \mathrm{SD}$ : mean \pm standard deviation

took place, all subjects received explanations about the purpose and procedure of this study in general, and agreed to participate voluntarily in the experiment. According to the Declaration of Helsinki, information on the study was provided, and the individuals gave their written consent to participate. The study was approved by the Institutional Review Board of Daegu University (No. 1040621201903-HR-010-02).

\section{Measurement}

The FTSST was used to assess the functional leg strengths of the subjects. This is a test that measures the time of a subject sitting in a chair while performing five sitting actions. The FTSST is a measurement of the independence, recurrence of falls, and status of daily living. In the case of elderly subjects, it also measures the functional aspects of strength within the lower extremities affected by conditions, such as cerebral palsy, strokes, and osteoarthritis [12]. Before starting the measurements, the therapists measured the subjects after demonstrating the test. Physical therapists instructed the subject to sit up five times as fast as they could and performed the measurement at the same time. During the course of the measurement, the therapists were careful not to record the use of their upper extremity power to support and stand up but instead only focus on their lower extremities. To avoid focusing on the measurement, the therapists did not speak during the performances. This measurement tool is a reliable tool for the elderly with an ICC $=.95$ test-retest reliability [12].

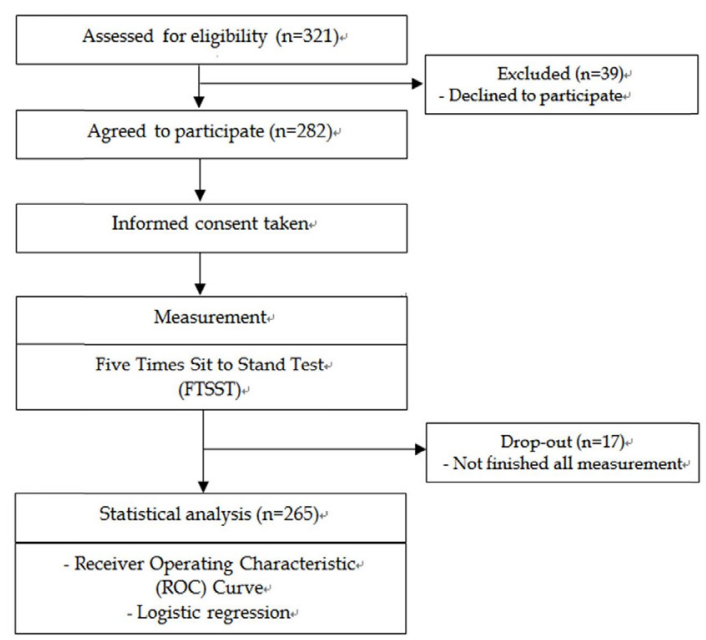

Fig. 1. Study protocol.

\section{Study Protocol}

Before the experiments were conducted, all subjects were explained the experimental methods and procedures. Because the subjects were elderly, safety observations were made during the measurements and the subjects were made to be uncomfortable. The FTSST was performed in the sitting position on a chair, and when the subjects complained of fatigue during the measurement, they were allowed to take sufficient rest. To prevent the fatigue of the leg muscles from affecting the other measurement results, the FTSST was measured on a different day and was not measured at a single time. The FTSST measurements were taken by two physical therapists. After a single measurement was made, the subjects were again scheduled to repeat the process and were re-measured on the other day. All measurements were taken three times and the results are expressed as the mean \pm standard deviation (Fig. 1).

\section{Statistical analysis}

Statistical analysis was performed using PASW Statistics software version 23.0 (IBM Co., Armonk, NY, USA). Descriptive statistics were used to obtain the general characteristics of the subjects. To examine the effects of 
Table 2. Effect of FTSST on a Risk of Dependent Daily Living

\begin{tabular}{cccccc}
\hline Variable & B & SE & Wald & $p$ & OR \\
\hline FTSST & .093 & .019 & 23.587 & $.000^{*}$ & 1.098 \\
\hline
\end{tabular}

${ }^{*} p<.05$

OR : Odds ratio

FTSST : Five times sit to stand test

Table 3. Optimal Cut-off Values to Predict the Risk of Dependent Daily Living

\begin{tabular}{ccccccc}
\hline Variable & Cut-off & Sensitivity & Specificity & $p$ & SE & ROC-AUC (95\% CI) \\
\hline FTSST & 15.62 & .626 & .753 & $.000^{*}$ & .034 & $.707(.640-.774)$ \\
\hline$* 0.05$
\end{tabular}

${ }^{*} p<.05$

AUC : Area under the curve

$\mathrm{CI}=$ Confidence interval

the FTSST on the independence of daily living, the subjects were classified into independent and dependent living groups according to their lifestyle, and their influence was then examined through logistic regression analysis. To determine the usefulness and cut-off value of the FTSST, the analysis was performed using the ROC curve (receiver operating characteristic). The cut-off values were set to the point where the sensitivity and specificity, the two selected variables, were greatest. Statistical significance was set at .05 .

\section{Results}

\section{Effects of the FTSST on lifestyle}

The elderly were more likely to live in a group rather than independently as the FTSST time increased $(p<.05)$ $(\mathrm{OR}=1.098)$. As the FTSST increased by one second, the probability of living in a group increased 1.098 fold (Table 2).

\section{The discrimination of FTSST on life style}

The area of the lower part of the ROC curve was .707, and a higher FTSST indicated, that a subject was more likely to live in the group rather than independently $(p<.05)$. The cut-off value was assigned to the point where both
Table 4. Coordinate of the ROC Curve of FTSST

\begin{tabular}{ccc}
\hline FTSST & Sensitivity & 1-Specificity \\
\hline 15.49 & .626 & .260 \\
15.57 & .626 & .253 \\
15.62 & .626 & .247 \\
15.69 & .617 & .247 \\
15.76 & .609 & .247 \\
15.96 & .600 & .247 \\
\hline
\end{tabular}

FTSST : Five times sit to stand test

the specificity and sensitivity were at the coordinates; the sensitivity and specificity were .626 and .753 , respectively, at 15.62 seconds (Tables 3, 4) (Fig. 2).

\section{Discussion}

The elderly tend to be less independent with regards to the functioning of daily living and change their lifestyle [7]. According to the survival of functional independence, the life style of the elderly was divided into the independent life and dependent group life [8]. Therefore, maintaining the functional independence of the elderly is an important factor in determining their lifestyle.

According to previous studies, a range of factors, such as decreased vision, cardiovascular diseases, and cognitive 


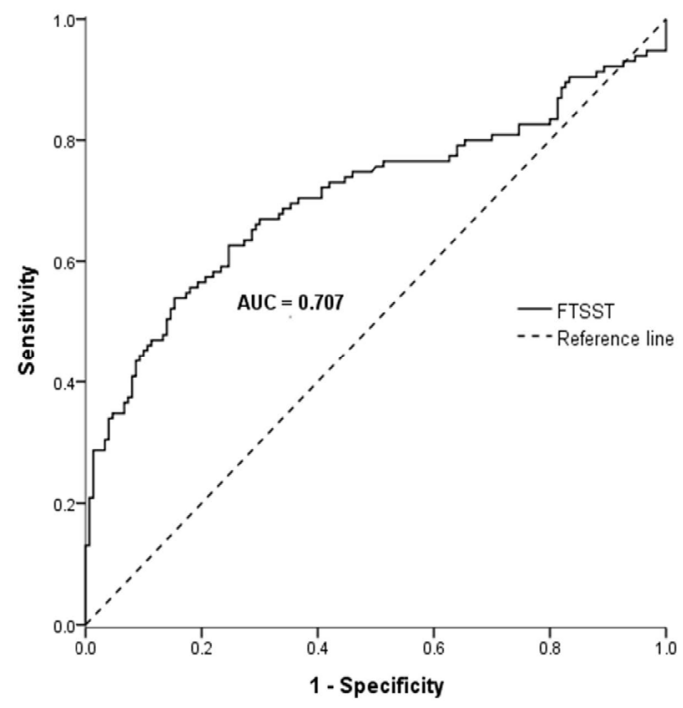

Fig. 2. FTSST ROC curve for the diagnostic test on the dependence of daily living.

impairment, affect the functional independence of the daily living performance, but this is strongly correlated with the muscular strength of the lower extremity muscles. Because the lower limbs support the body and movements, such as walking, weakness of the lower limbs reduce the stability and mobility, resulting in falls, decreased functional independence, and a change to a dependent lifestyle [13]. In this study, the functional independence of the daily living performance ability was predicted by evaluating the leg strength using the FTSST. The FTSST is a method of assessing the leg strength by measuring the time it takes to complete a sitting task five times in a chair; the test-retest reliability of the elderly was $\mathrm{ICC}=.597$, which is a highly reliable measure. In addition, the prediction accuracy of functional independence was reported to be high, and a simple evaluation can be performed on the elderly [14]. This paper presents the baseline values of the FTSST measurement on the functional independence of elderly people in Korea.

A higher FTSST indicated a higher likelihood that they would be dependent on a group lifestyle rather than living independently. The odds ratio, cut-off value through the
ROC curve, sensitivity, and specificity were $1.098 ; 15.62$ seconds, .626, and .753 , respectively. The area of the lower part of the ROC curve was .707 , which was greater than .5 , and the accuracy was fair. This suggests that the probability of living in a group increases 1.098 fold as the FTSST increases by 1 second, based on 15.62 seconds for elderly people.

In a previous study conducted on subjects with an average age of 70 years, the cut-off value showed low sensitivity and high specificity based on 15 seconds [15]. In addition, studies of elderly people over 65 years of age showed normal sensitivity and specificity for the FTSST at 15 seconds, and increased risk of falls and decreased functional independence when the time was more than 15 seconds [16]. This is consistent with the results of the present study. In addition, the sensitivity and specificity are often used to assess the accuracy of an assessment that predicts the outcomes. Sensitivity refers to how well people are actually identified when they are tested, and the specificity means how well they classify those who are not. In general, the accuracy of the assessment can be measured by the AUC (area under the ROC curve) using the sensitivity and specificity, and it can be measured as non-informative $(\mathrm{AUC}=.5)$ or less accurate $(.5<\mathrm{AUC} \leq .7)$ $(\mathrm{AUC}=1)$, which is still very accurate $(.9<\mathrm{AUC} \leq 1)$ [17]. In this study, the AUC value was .707, which was moderately accurate. The cut-off value in this study showed a higher sensitivity and specificity compared to previous studies. This means that the accuracy of the FTSST evaluation tool for predicting the functional independence of the elderly is high and the results of this study are acceptable. In addition, this topic was conducted previously on foreigners in another study, but this study is more meaningful in that it showed high sensitivity and specificity in the observation of elderly people in Korea.

Previous studies reported the typical values of the FTSST in subjects in their $60 \mathrm{~s}, 70 \mathrm{~s}$, and $80 \mathrm{~s}$ to be 11.4 , 12.6, and 14,8 seconds, respectively. In a study of those aged between 74-98, the cut-value was higher than 13.6 
seconds, and the risk of falls and disability were increased significantly $[12,15]$. In addition, cut-off values in the study of the elderly, with a mean age of 80 years, showed a normal sensitivity and specificity at 12 seconds [18]. This was faster than the results of the present study and did not match. In other words, when the previous studies were combined, the cut-off values of the FTSST ranged between 12 and 15 for the functional independence predictions, and the sensitivity and specificity were also different. Therefore, it is necessary to carry out multiple studies to determine the optimal cut-off value.

In addition, previous studies evaluated the ankle, knee, and hip joint muscle strength of the elderly through the Manual Muscle Test (MMT). An increase in lower extremity muscle strength indicates a higher likelihood of living independently. The previous results are believed to support the results of the present study [19].

The results of this study suggest that the elderly in Korea are more likely to live a group-dependent lifestyle rather than live independently; the likelihood of this outcome is increased further for every additional second beyond 15.62 seconds. In this study, the loss of independence of daily life could be predicted based on the status of a subject's lower leg strength using the FTSST. The data collected from this tool can be used as a reference and numerical values for prevention. In addition, increasing the strength of the lower limbs should be considered for improving the independence of the elderly. Furthermore, this study is more meaningful because it is a study on Korean elderly people, and the numerical value of the elderly people in Korea will be analyzed more accurately if this study is expanded in the future. This study can lead to improved elderly life styles, diversification of elderly studies, and enhanced quality.

The limitations of this study were as follows. First, the age range of the subjects was higher than that of general subjects. In future studies, it will be necessary to study the baseline values according to specific age groups.
Second, it is not comparable with various evaluation tools. The cause of falls as a single predictor is difficult to explain because of the various complex causes of ADL. Future research will be needed to combine the various predictors of $\mathrm{ADL}$

\section{Conclusion}

The aim of this study was to provide the standard value of the FTSST measurement on the daily life independence of the elderly in Korea and examine the effects on their daily lives. The results suggest that the elderly in Korea are more likely to live a group-dependent lifestyle rather than live independently; the likelihood of this outcome is increased further for every additional second beyond 15.62 seconds. The data collected from this tool can be used as the reference and numerical values for prevention. In addition, increasing the strength of the lower limbs to improve the independence of the elderly should be considered.

\section{Acknowledgments}

This study was supported by Uiduk University Foundation Grant, 2018.

\section{References}

[1] Ministry of Government Administration and Home Affairs. Resident registration demographics. 2017

[2] National Statistical Office. 2012 Statistics for the elderly. 2011.

[3] Hannan MT, Felson DT, Dawson HB, et al. Risk factors for longitudinal bone loss in elderly men and women: the Framingham Osteoporosis Study. J Bone Miner Res. 2000;15(4):710-20.

[4] Hurley MV, Rees J, Newham DJ. Quadriceps function, proprioceptive acuity and functional performance in 
healthy young, middle-aged and elderly subjects. Age ageing. 1998;27(1):55-62.

[5] Miller PA, Butin D. The role of occupational therapy in dementia-COPE (Caregiver Options for Practical Experiences). Int J Geriatr Psychiatry. 2000;15(1):86-9.

[6] Andersen CK, Wittrup-Jensen KU, Lolk A, et al. Ability to perform activities of daily living is the main factor affecting quality of life in patients with dementia. Health Qual Life Outcomes. 2004;2(1):52.

[7] Rochat S, Cumming RG, Blyth F, et al. Frailty and use of health and community services by community-dwelling older men: the Concord Health and Ageing in Men Project. Age ageing. 2010;39(2):228-33.

[8] Covinsky KE, Palmer RM, Fortinsky RH, et al. Loss of independence in activities of daily living in older adults hospitalized with medical illnesses: increased vulnerability with age. J Am Geriatr Soc. 2003;51(4):451-8.

[9] Moreland JD, Richardson JA, Goldsmith CH, et al. Muscle weakness and falls in older adults: a systematic review and meta analysis. J Am Geriatr Soc. 2004;52(7):1121-9.

[10] Schenkman M, Hughes MA, Samsa G, et al. The relative importance of strength and balance in chair rise by functionally impaired older individuals. J Am Geriatr Soc. 1996;44(12):1441-6.

[11] Andrews AW, Thomas MW, Bohannon RW. Normative values for isometric muscle force measurements obtained with hand-held dynamometers. Phys ther. 1996;76(3): 248-59.
[12] Bohannon RW. Reference values for the five-repetition sit-to-stand test: a descriptive meta-analysis of data from elders. Percept Mot skills. 2006;103(1):215-22.

[13] Fiatarone MA, Evans WJ. 11 The Etiology and Reversibility of Muscle Dysfunction in the Aged. J Gerontol. 1993;48:77-83.

[14] Tiedemann A, Shimada H, Sherrington C, et al. The comparative ability of eight functional mobility tests for predicting falls in community-dwelling older people. Age ageing. 2008;37(4):430-5.

[15] Carter V, Jain T, James J, et al. The 3-m backwards walk and retrospective falls: diagnostic accuracy of a novel clinical measure. J Geriatr Phys Ther. 2019.

[16] Buatois S, Perret-Guillaume C, Gueguen R, et al. A simple clinical scale to stratify risk of recurrent falls in community-dwelling adults aged 65 years and older. Phys Ther. 2010;90(4):550-60.

[17] Greiner M, Pfeiffer D, Smith RD. Principles and practical application of the receiver-operating characteristic analysis for diagnostic tests. Prev Vet Med. 2000; 45(1-2):23-41.

[18] Tiedemann A, Lord SR, Sherrington C. The development and validation of a brief performance-based fall risk assessment tool for use in primary care. J Gerontol A Biol Sci Med Sci. 2010;65(8):896-903.

[19] Kim SG. The Effects of Risk Factors for Falls Based on the Framework of ICF on the Lifestyles of the Elderly. Doctor's Degree. Daegu University. 2014. 